CAHIERS DE

NARRATOLOGIE

\section{Cahiers de Narratologie}

Analyse et théorie narratives

17 | 2009

Stéréotype et narration littéraire

\title{
Nosotras que no somos como las demás de Lucía Etxebarria : stéréotype contre stéréotype
}

\section{Christine Di Benedetto}

\section{OpenEdition}

Journals

Édition électronique

URL : http://journals.openedition.org/narratologie/1223

DOI : 10.4000/narratologie.1223

ISSN : $1765-307 X$

Éditeur

LIRCES

Référence électronique

Christine Di Benedetto, « Nosotras que no somos como las demás de Lucía Etxebarria : stéréotype contre stéréotype », Cahiers de Narratologie [En ligne], 17 | 2009, mis en ligne le 17 décembre 2009, consulté le 01 mai 2019. URL : http://journals.openedition.org/narratologie/1223 ; DOI : 10.4000/ narratologie. 1223

Ce document a été généré automatiquement le 1 mai 2019.

\section{c) (i)}

Cahiers de Narratologie - Analyse et théorie narratives est mis à disposition selon les termes de la licence Creative Commons Attribution - Pas d'Utilisation Commerciale - Pas de Modification 4.0 International. 


\title{
Nosotras que no somos como las demás de Lucía Etxebarria : stéréotype contre stéréotype
}

\author{
Christine Di Benedetto
}

1 Nosotras que no somos como las demás ${ }^{1}$ de la romancière espagnole Lucía Etxebarria s'inscrit dès son titre, "Nous, qui ne sommes pas comme toutes les autres", dans le concept d'altéritée . C'est lui qui guide la lecture en installant comme horizon d'attente celui de l'affirmation identitaire. Dès lors sont figurés deux groupes antagonistes dont l'œuvre se charge de faire apparaitre les traits. De l'opposition entre ces collectifs potentiellement identifiables découle le sens de l'oeuvre, sorte de positionnement axiologique dans l'Espagne du troisième millénaire. Qui sont précisément ce «nous » et ces «autres »? Comment s'installe la relation entre les deux? Quelle identité et quel reflet de société s'esquissent dans ce combat? Il s'agit, dans le titre, de mettre en présence deux groupes féminins : «nous » et « toutes les autres » (nosotras, las demás). Et d'entrée, de les opposer, car l'existence du nouveau groupe s'inscrit dans la négation, au niveau de l'essence même - " être ", qui est rendu par le verbe ser - ce que les autres ne sont pas. C'est à travers la notion de stéréotype, dont nous cherchons ici à analyser les enjeux et les formes dans la narration, que nous proposons d'entreprendre le décryptage du chemin que doit effectuer le lecteur pour identifier ces groupes.

2 La question générique n'est pas anodine puisqu'on se voit recommander explicitement dans le prologue de lire ce livre «comme s'il s'agissait d'un roman ${ }^{3}$ ». Ce «comme si » interpelle... Formellement l'oeuvre se compose d'éléments divers. S'enchaînent sur 396 pages un prologue, une liste de Dramatis personae présentant quatre personnages féminins, 16 chapitres avec titre et enfin un index, qui reprend le titre des chapitres en les numérotant et faisant figurer leur personnage principal. On a la double sensation d'une profusion d'éléments informatifs et d'une volonté d'imposer une cohérence à l'ensemble, dans un élan argumentatif à peine déguisé. 
Les chapitres portent tous des titres étranges, en latin ou latinisés, ce qui les rend à première vue opaques. Parfois ils demeurent mystérieux, comme le 5. Alexitimia ou le 7. Visio Smaradigna, Le plus souvent au contraire ils apparaissent clairement figuratifs derrière une légère déformation linguistique, par exemple le 10. Vagina Dentata, le 14. Sed non satiata, le 15. Nocturnalia... Le Prologue s'attache à expliciter certains d'entre eux. Il montre leur spécificité, insistant sur le fait qu'il s'agissait de nouvelles séparées à l'origine, ayant chacune une genèse spécifique : "au début, c'était un livre de récits ${ }^{4} . .$. », histoires de vies qui ne se croisent pas. Initialement prévu pour être un livre de nouvelles ou de textes de commande indépendants, le projet a été modifié par la nécessité dont fait état l'auteur de laisser un espace suffisant pour que les personnages isolés puissent se rencontrer, échanger des expériences, et, structurellement, à partir d'individualités et d'expériences diverses, trouver une consistance en tant que groupe dans leurs traits communs. Ainsi prend naissance la notion de collectif dans lequel s'inclut l'auteur dès le prologue, mais qui doit acquérir progressivement sa consistance d'univers féminin en lutte.

Les individualités, auxquelles est confiée la charge de dessiner dans la diégèse le théâtre de la vie dans un contexte espagnol, figurent dans la « Dramatis Personae ». Cette liste ne comprend que quatre personnages, tous des femmes, entre 25 et 31 ans. Elles évoluent d'une nouvelle à l'autre, accompagnées occasionnellement $(12,13,14,15)$ de deux personnages masculins, Jaime et Eduardo. Même la plus âgée est une enfant de la fin de la dictature franquiste puisqu'on peut établir son acte de naissance à 1968. Toutes sont adultes vers l'an 2000. Comme la majorité des jeunes espagnoles abordant le $\mathrm{XXI}^{\circ}$ siècle, elles disposent d'une formation et d'un diplôme universitaires : Beaux Arts pour Raquel, Philologie anglaise pour Elsa, Droit pour Susi, Commerce pour María. Comme beaucoup d'entre elles, si l'on fait exception du chômage qui les touche en priorité et qui ne relève pas d'un choix personnel, elles poursuivent une carrière professionnelle : respectivement mannequin, traductrice-journaliste, conseillère fiscale et responsable marketing. L'âge, les études et la profession sont les trois seuls axes selon lesquels sont présentées ces héroïnes. Ni patronyme ni statut affectif ni situation de famille ne les rattachent sous une forme généalogique à un passé ou à un avenir. Ces quatre personnages de femmes sont destinés, par leur richesse, à configurer tout un univers féminin, à l'instar de María : « des infinités de Maria qui composaient tout le spectre définissable des personnalités féminines (...) seule avec elle-même, ou avec des nous en elle ${ }^{5}$ ».

Le titre du roman, la liste de personnages, l'insertion de la romancière - par la première personne du pluriel, le «nous» évoqué ci-dessus - dans le collectif de ces femmes qui vont évoluer tout au long du roman, sont autant d'éléments qui semblent mener vers la mise en place d'un affrontement femmes-hommes. En effet parmi les stéréotypes auxquels on peut s'attendre, le stéréotype de genre s'installe souvent, à partir d'un éternel féminin ou masculin, accepté ou rejeté.

6 En outre, comme le suggère Angenot qui parle "d'idéologème dialogique et polyphonique $^{6}$ ", si l'on retient l'importance de la «contextualisation", l'œuvre d'Etxebarria dans son ensemble est largement marquée par le combat féministe. En outre du fait de «l'interdiscursivité " d'une période donnée, dans l'ère géographique hispanique contemporaine les stéréotypes liés à l'histoire des mentalités et fondés sur des réalités historiques se greffent sur ce stéréotype de genre. Une bonne partie du chapitre 7, Visio Smaradigna ${ }^{7}$, est consacré au visionnage par deux amies de cassettes vidéo érotiques. Supports pour initiés, pourrait vouloir dire le titre, mais en réalité simplement 
faux guides d'apprentissage sexuel pour couples, explique l'auteur dans le Prologue p.16. L'expression "porno softcore" pour femmes, instaure une hiérarchie dans le public supposé, les femmes semblant tenues à l'écart d'une "vraie » pornographie, celle du " montrer tout ", qui serait réservée aux hommes. Le chapitre lui-même, avec les détails de chacune des cassettes et les critiques avisées qu'en font Elsa et Raquel, prouve que la distinction entre les récepteurs est infondée. L'hypocrisie annoncée dans le projet, éviter aux femmes «l'humiliation de se rendre dans un sex-shop (territoire masculin, le plus souvent $\left.{ }^{8}\right)$ » se retrouve dans le chapitre où le ton est ironique. La critique porte sur la bipolarisation des attitudes face à la sexualité et ce genre de produit, dont l'existence et l'utilisation seraient mal assumées par les femmes, en est emblématique. On y trouve par ailleurs une définition de stéréotypes de genre qui diffèrent en Espagne et aux Etats Unis : «Le critère de sélection des personnages de ce type de cassettes vidéos relevait de canons scandaleusement nord-américains dont Raquel doutait vraiment qu'ils puissent correspondre aux goûts franchement locaux d'Elsa ${ }^{9}$ ».

7 Jusque là, tout semblerait aller dans le même sens d'opposition homme/femme, mais la vision va s'affiner.

Certes le Prologue inscrit l'œuvre dans la perspective de la différence dès la première phrase, d'autant que celle-ci est remarquable parce qu'inachevée et isolée du corps du texte: «Ils ne sont pas comme les autres ${ }^{10} \ldots$ ». Toutefois la reprise des principaux éléments du titre mais en y insérant le masculin a de quoi étonner. S'ensuit une réflexion sur le mépris et le rejet que déclenchent encore aujourd'hui les tentatives de remise en question des modes de pensée et d'action traditionnels, ce qui donne au lecteur la sensation de lire un essai. Ce rejet est même analysé, considéré comme normal « dans les moments de changement culturel»(p8), ce qui est le cas dans l'Espagne résolument démocratique et européenne de la fin du deuxième millénaire, que Lucía Etxebarria choisit comme cadre de ce roman publié en 1999. Clairement aussi, l'axe selon lequel est examiné ce changement est celui des relations hommes-femmes puisque l'un et l'autre sont présentés comme inextricablement liés, tant au niveau social -« les rôles que nous adoptons ${ }^{11} »-$, qu'au niveau individuel - notre identité sexuelle - ce que signifie, pour nous, être homme ou femme ${ }^{12}$ ». Des allusions à des disciplines scientifiques comme l'anthropologie ${ }^{13}$, la présentation des différents mouvements féministes, l'étude statistique de la place des femmes dans le monde du travail semblent faire état de faits qui justifieraient le clivage entre celles qui acceptent ou rejettent ces rôles établis par des années de système patriarcal et de contrôle des moeurs. Mais pas seulement celles, dit Etxebarria, celles et ceux...

Partant de cela, le groupe, ou l'endogroupe, comme l'appelle Henri Tajfel ${ }^{14}$ dans ses travaux parus entre 1969 et 1979, et en particulier dans sa théorie de "l'identité sociale ", commence à se constituer, tantôt au masculin de généralité, le plus souvent au féminin, autour de la notion de revendication. Sur moins de deux pages est déclinée une série de huit verbes à la première personne du pluriel, tous employés dans une structure figée. L'expression "algunas mujeres ", certaines femmes ou bien certaines d'entre nous, est suivie d'un verbes de rejet ou employé sous forme négative: «nous ne sommes pas d'accord..., nous n'aimons pas..., nous en avons $\operatorname{assez}^{15} . . . »$. Dans un contexte d'antagonismes attendus entre femmes et hommes, l'accentuation des similitudes entre membres d'un même groupe va progressivement relever du stéréotype. Celui-ci a une première fonction dans la vie sociale, agir sur la cohésion d'un groupe. Il lui permet en effet d'abord de montrer sa différence. Même si certains hommes peuvent en faire partie, 
il s'agit d'abord d'évoquer ici un groupe constitué par certaines femmes face à d'autres femmes, comme si la catégorie féminine était scindée. Apparaît alors une première définition de ce qu'on pourrait appeler un groupe actif à partir d'une affirmation, isolée du reste du texte : « certaines d'entre nous, nous protestons ${ }^{16}$ ».

Dans un deuxième temps donc, la constitution de ce groupe permet de se valoriser au détriment des autres, l'exogroupe déjà existant, le groupe de celles qui ne protestent pas et qui apparaît fortement marqué et largement stéréotypé. L'endogroupe qui se construit ici serait donc constitué de femmes, anonymes, ou identifiées (liste p.21) à qui ce livre se veut explicitement dédié, comme il pourrait l'être à des hommes qui ne seraient pas non plus comme les autres. De fait, chez Lucía Etxebarria, les autres ne sont pas les hommes, mais les femmes, ou les hommes, qui n'ont pas évolué. Dans ce roman, nous constatons que les systèmes sociaux produisent les stéréotypes dont ils ont besoin à la fois pour se perpétuer et pour se légitimer eux-mêmes. La question réside donc bien dans la différence, mais où on l'attendait moins, entre les femmes.

Lucia Etxebarria invite donc à lire son roman comme un manifeste. Et elle le fait à travers un jeu de stéréotypes. Elle met en évidence l'existence de deux groupes de femmes que le récit configure, mais surtout le refus du second de ressembler au premier. La priorité est donc d'établir comment sont constitués ces groupes en voyant en quoi consiste l'ordre établi, à partir du moment où certains des membres dérogent à une pratique, voire la transgressent volontairement. Car selon elle, le texte littéraire est un moyen pour l'être humain de combattre un stéréotype qui domine dans la vie quotidienne, qui est gravé dans les consciences et imprègne toutes les productions culturelles.

Lorsqu'on prend le cas de la femme qui accepte de perpétuer une situation existante, entre en jeu le puissant stéréotype qui lie la définition de la femme à sa soumission à l'homme ${ }^{17}$, puisqu'il est généralement considéré qu'un stéréotype est d'autant plus fort qu'il est réducteur et pauvre en informations. Lucía Etxebarria se propose d'utiliser les mêmes vecteurs avec un stéréotype inverse ${ }^{18}$.

13 Comment s'exprime donc, dans le récit romanesque, cette volonté à présent claire de la part de l'auteur de tenter de dénoncer les stéréotypes traditionnels de la femme? Et comment s'organise ici la narration pour inscrire un nouveau stéréotype sur ces "femmes qui ne sont pas comme les autres", et par là même apporter une valeur négative à l'existant, celui qui s'applique aux « autres» en question? C'est ce que nous allons chercher à comprendre à présent.

Réfléchir à la notion de stéréotype nous demande de l'envisager sous l'angle de sa fonction puis de son mécanisme. Envisager sa fonction c'est voir comment un stéréotype, qui prend forme dans ce roman, peut être à même de s'imposer à côté ou au-dessus de celui qui dominait à un moment donné. Lucía Etxebarria écrit pour un public en quête ou en attente de modèle. Comme elle le dit dans son Prologue, le chapitre 12, Stigmata, est la clé de lecture du roman. A la page 295, Raquel pose en effet qu'il existe deux types de femmes : de catégorie A ou B. Et l'enjeu est annoncé :

si tu es dans la seconde, tu seras le centre d'une polémique constante. La moitié du monde ta haïra, te crucifiera pour la moindre action et fera de toi la cible de ses craintes, ses haines et son mépris ${ }^{19}$.

15 A ce propos, la féministe Antoinette Fouque défend l'idée que :

l'avenir sera ce que les femmes qui ont trente ans aujourd'hui feront de leur héritage. (...) Nos filles, si elles ont le courage de compter sur leurs propres forces, pourront compter 
sur elles et sur les hommes libérés de la peur, de l'envie, les hommes de bonne volontée ${ }^{20} \ldots$ quatre héroïnes de trente ans et de toutes les filles de catégorie B, présentées ici en guise de provocation sous les traits négatifs que leur prêtent, dans le vocabulaire même, celles de la A :

Les filles faciles, les garces, les putains, celles qui volent les maris, celles qui comme moi ne s'occupent pas d'enfants, entrent et sortent quand et par où elles en ont envie... et qui ne portent pas de bague avec une date dedans ${ }^{21}$. "comparaison sociale" entraîne des attitudes de discrimination nécessaires dans la formation de l'estime de soi qui est refusée par le langage couramment employé de " putain » ou de " garce».

18 Si l'on entend le stéréotype comme une représentation sociale partagée, comme un élément relativement fixe et stable pour la représentation des autres et son autoreprésentation en matière d'identité, on ne peut prétendre qu'il soit créé en un seul roman. La sociocritique voit dans le stéréotype la « rumeur anonyme d'une société et de ses représentations ${ }^{22}$ ». Il est filtre et trace, dans le texte littéraire, de la socialité. Nosotras que no somos como las demás relaie et précise cette rumeur. Lucía Etxebarria en créant un monde vraisemblable de lieux et de personnages, démystifie petit à petit les valeurs de la société qui l'abrite. Barthes quant à lui considère que le stéréotype participe de la doxa: «La Doxa (mot qui va revenir souvent), c'est l'Opinion publique, l'Esprit majoritaire, le Consensus petit-bourgeois, la Voix du Naturel, la Violence du Préjugé23 ». Le point commun de la «doxa» et de la lecture sociocritique des stéréotypes se trouve dans la reconnaissance de l'historicité et de la socialité du langage et des textes, ce qui ici est largement sous-tendu, nous l'avons vu, par un Prologue qui oscille entre féminisme militant et tentative de nuancer, en incluant les hommes dans les deux catégories. «A la croisée des sciences sociales et des études littéraires, il (le stéréotype) se définit comme une représentation sociale, un schème collectif figé qui correspond à un modèle culturel daté ${ }^{24}$ », la prise en main d'un modèle de société où la rébellion contre l'existant est possible.

Intéressons-nous à présent aux représentations sociales à l'œuvre dans la fiction, aux modes de présentation de l'évidence qui contribuent à la réaffirmation des idées reçues, à la problématique du discours qui mêle critères formels, énonciatifs, thématiques et historiques. Lucía Etxebarria s'attelle à configurer le stéréotype assumé de la femme « autre », rebelle, et vise à faire de lui un «ferment actif», comme le dit Ruth Amossy. Il s'agit pour elle en même temps de ne pas tomber dans le piège du cliché mal vécu, en particulier parce qu'elle installe ce stéréotype directement en opposition avec un autre. La reprise et la modulation du stéréotype par le discours se fait ainsi de manière originale. La prise en charge est double : stéréotype attaché à l'endogroupe (noté sEn, celles qui protestent) et stéréotype attaché à l'exogroupe (noté sEx, celles qui acceptent) et chacun connaît un traitement différent. Ce qui nous occupe est de voir se mettre en action justement «ce jeu avec la croyance qui s'établit entre le texte et les représentations qu'il met en œuvre ${ }^{25} »$.

John Turner, qui a travaillé avec Henri Tajfel, a montré comment la problématique de groupe s'inscrit dans le cadre d'un processus de compétition sociale. Mais aussi que 
comme chaque groupe a cette même démarche, il s'ensuit une sorte de compétition des groupes pour l'identité positive qui entraîne une situation de rivalité. Ici l'auteur ne donne la parole qu'à l'endogroupe. Les traits du stéréotype qui le définit occupent la plus grande place. De fait l'autre, l'existant, n'est qu'implicitement évoqué par antiphrase ou obliquement mentionné.

21 En outre, s'agissant ici d'un stéréotype en devenir, la fonction constructive du sEn est plus visible puisqu'on ne le considère plus de façon statique avec des contenus et formes figés, comme le sEx. Au contraire, il prend son intérêt dans le sens où un individu, l'auteur, ou bien un groupe -ces femmes qui ne sont pas comme les autres- se l'approprient et l'intègrent dans une dynamique entre soi et les autres. Aux portes d'un territoire encore peu exploré, c'est la variété des possibles qui caractérise cet endogroupe, pas la sécurité : « la recherche vaut, en elle-même, la peine ${ }^{26}$ ».

22 Les critères qui le définissent sont peu nombreux et évoquent surtout le rapport au corps, en particulier la sexualité. Ils relèvent à la fois de ce que John Turner appelle représentations « justificatrices » et " anticipatrices ${ }^{27}$ ». La liberté sexuelle est le maître mot et c'est en elle que les différents personnages féminins connaissent à tour de rôle «l'expérience la plus révélatrice de leur vie ${ }^{28}$ ». Le roman dissocie amour et désir, mais associe ce dernier à la sexualité, devenue érotisme exacerbé, comme il élargit la sexualité au-delà des rapports homme/femme.

23 L'amour est vécu dans l'absence (par exemple, la mort par noyade du frère aimé dans le chapitre 1). Incapacité définitive à partager, il est perçu comme menant sur le vide. Et l'on y découvre, renversant tous les stéréotypes de romantisme ou de sentimentalisme attachés à la vision de la femme, une perversion redoutable quand « on finit par aimer ce que l'on craint ${ }^{29}$ ", avec la certitude que "en amour, comme à la bourse, plus tu investis plus tu perds ${ }^{30} »$. Pour autant, la réflexion sur l'amour n'est pas absente, mais aucune conclusion ou recette n'est promise pour trouver cette chose, « aussi inexplicable que le sexe des anges ${ }^{31} »$.

24 En revanche, concernant la sexualité, toutes les formes sont évoquées sans aucun rejet a priori, ni crainte brandie d'une possible « addiction au sexe »(p.40-42-179). Cela semble le critère le plus significatif de ce nouveau groupe; de fait le récit introduit de très nombreuses scènes de sexe. Celui-ci présente deux versants : le côté mécanique dans le cadre de l'exogroupe devient une possible transcendance dans la subversion, offerte au seul endogroupe. Les frontières se brouillent alors et de manière provocatrice, les éléments de la pratique religieuse sont appliqués à la sexualité, dans un renversement total des valeurs. Des termes relevant de deux champs lexicaux traditionnellement opposés sont associés pour évoquer un amour physique fait de "prières hérétiques, rites profanes, baptêmes sacrilèges de sueur et de halètements ${ }^{32}$ ». La compréhension du concept fondateur du christianisme, dont la version catholique a constitué la base de la société espagnole pendant quatre décennies, est même rendue possible par une sexualité jusqu'alors reçue comme amorale, la relation d'une femme avec deux hommes: "l'addition de deux et un ne faisait pas trois. María avait compris, enfin, pourquoi la Trinité est comprise comme un $\mathrm{Un}^{33}$ ». Le parallèle va jusqu'à l'extase mystique et la résurrection de la chair dans « un don de sa chair pour la faire renaître, pour défier sa condition de mortel ${ }^{34} »$. Ce sexe-là, dans une autre combinaison, celle d'un des deux hommes, Grahame, avec Maria, a même une force primaire, tellurique : «l'un contre l'autre ils réinventèrent l'explosion initiale de laquelle avait surgi le $\operatorname{cosmos}^{35} »$. Il n'y a donc pas négation de l'homme, ni avilissement dans l'acte, mais cela ne conduit pas pour 
autant à la vie à deux. Le sexe devient métaphore de la vie, «toujours le même, toujours différent, comme chaque jour, comme la vie ${ }^{36}$ ». Par conséquent, il n'y a aucune surprise dans le fait de rencontrer des situations très diverses : sexualité accomplie (chap. 7) ou simple attirance physique (chap. 16) entre filles ou rapports extra-conjugaux avec un gigolo (chap. 13). La femme met en œuvre ses choix, parachevant l'inversion des rôles par rapport à l'attente machiste de l'homme-étalon : « quelque chose se dégonfla à l'intérieur d'Eduardo lorsqu'il comprit qu'en réalité, c'était elle qui l'avait dirigé tout le temps ${ }^{37}$ ».

L'opposition entre les deux groupes se confirme par l'association de termes positifs autour d'un mot toujours connoté négativement, la "dépravation ». Ainsi le fantasme sado-masochiste devient une force parce qu'il est devenu concevable : « elle commençait à comprendre à quel point la dépravation est facile et sûre lorsqu'on a trouvé le courage de commencer ${ }^{38} »$.

Parmi les éléments subversifs, il en est un autre, la drogue, qui se glisse légèrement dans le texte comme une évidence, pour évoquer une ambiance : «le nuage plein d'empathie du haschich » (p.79), ou décrire un personnage, « sylphide squelettique... les yeux drogués entrouverts ${ }^{39} »$. Il n'est pas non plus présenté comme une solution mais confirme le refus d'accepter une barrière extérieure, quelle qu'elle soit.

La définition des caractéristiques de l'endogroupe se fait aussi « en creux ", c'est-à-dire que certains critères attendus du fait des stéréotypes attachés à l'exogroupe sont remarquables par leur absence, c'est ce qui relève des représentations « induites ». Il n'y a rien dans ce roman concernant l'éducation que donneraient les quatre héroïnes à d'hypothétiques enfants. Ce rôle, pourtant traditionnellement dévolu à la femme ${ }^{40}$, n'est qu'effleuré par le biais des erreurs commises par leurs propres parents, nous le verrons plus loin. On ne trouve nulle part non plus de relation de couple stable parce que celui-ci est perçu comme fondamentalement inacceptable dans sa permanence: "jamais elle ne parviendrait à équilibrer (...) les exigences rivales de s'aimer elle-même et d'aimer les autres, l'angoisse de l'oppression et l'angoisse de l'abandon ${ }^{41}$ ». Et c'est ce niveau de compréhension, difficile à atteindre pour les quatre personnages, dans la dé-protection par rapport aux normes sociales, qui est la caractéristique de la femme différente. Donc en filigrane inversé, tout le vécu familial est rejeté, alors que, ou plutôt parce que, il est la base de l'autre groupe.

28 Le stéréotype lié à l'exogroupe, est formé, on l'a compris dans ce jeu d'opposition entre stéréotypes, par des éléments qui s'opposent point par point aux axes du précédent. Le sexe, revendiqué là-bas, est cliché ou nié ici. Dès la naissance de l'enfant, il y a marquage des places : le bleu habille le frère et le rose la soeur ${ }^{42}$. Mais ce qui caractérise la notion d' exogroupe c'est le jugement de valeur négatif qui y est attaché. A chaque signe du stéréotype correspond une critique plus ou moins implicite. La différenciation censée définir les rôles au sein de la famille ${ }^{43}$ et dans les relations garçon-fille, éveille paradoxalement l'amour incestueux en la petite fille qui devient femme face à l'homme qu'est son grand frère. Cette sexualité-là, rêvée mais interdite, est socialement tue, bien entendu. Le lecteur n'a accès qu'au point de vue de la jeune adulte qui se revoit enfant, avec ce seul espace de liberté en elle, et qui, adulte, va nager jusqu'à presque se noyer pour retrouver ce frère aimé et mort en mer. A côté de cette relation sublimée parce voulue, se dresse l'autre, haïe, que les parents facilitent, par leur absence et en voulant obstinément ignorer la situation, l'inceste du père sur sa fille de huit ans. Susi évoque la crainte, les cris, les sueurs froides, la perte de repères, la honte et en plus, le silence obligé (p.40-41). De fait, la famille, étendard suprême brandi par ceux et celles qui 
perpétuent le système, est écrite dans tous ses échecs. L'hypocrisie dans la famille est la marque d'une société ankylosée. Susi sait que pour des gens comme ses parents, on se marie et on a des enfants par habitude ${ }^{44}$. Par obligation aussi, parce que cette institution est la seule qui puisse autoriser la sexualité pour la femme dont le bien ultime est la virginité : «il dut épouser la mère pour pouvoir coucher avec elle (parce qu'elle arriva vierge à son mariage)... le seul acte dont elle pouvait se sentir fière dans sa vie ennuyeuse et stérile ${ }^{45} \%$. Logiquement alors, l'idée de couple se voit réduite à une sexualité technique ${ }^{46}$ que refuse la fille voulant se différencier de sa mère, et à l'inévitable conflit ${ }^{47}$. La simplification crue ("gymnastique de l'amour et vaisselle jetée à la tête ») dénote un pessimisme total devant des relations sans avenir et sans issue, basées sur le rapport de force. Si elle est échec pour les conjoints, la situation familiale n'est pas plus réussie pour les enfants. Aucune marque d'affectivité ("la mère", "le père", sans le possessif affectueux), de surveillance, d'échange, ni même de présence. La mère, figure perpétuellement en dépression nerveuse, silencieuse et enfermée dans sa chambre (p.44) ou au mieux dans sa cuisine, devenue sa marque, "son territoire, son royaume", la représentation du féminin dans son versant nourricier. Au contraire, l'enfant, au nom duquel la famille est supposée continuer à fonctionner, se voit contraint de mendier un amour refusé à l'avance. La sentence arrive sous forme de maxime sans appel : " qu'est la famille, qu'est l'enfance, en fin de compte, si ce n'est un tortueux entraînement au masochisme ${ }^{48}$ ». La famille, dans tous ses stéréotypes castrateurs et négatifs, est présentée p.182 dans un parallèle entre amour de famille et de couple. Dans les deux cas, l'individu est supposé aimer des gens dont il sait qu'ils ne lui rendront pas la pareille, par manque de temps ou parce qu'ils le culpabilisent en disant qu'il ne mérite pas cet amour car il n'aurait pas fait ce qu'il faut. Le narrateur, traduisant les pensées du personnage de Raquel, se fait l'écho des réflexions du philosophe «misogyne " Kirkegaard dans sa démonstration de la relation indissociable entre l'amour et l'angoisse, qui invaliderait définitivement ce fonctionnement et dont la seule explication serait « une addiction, une dépendance par rapport à l'angoisse, un besoin désespéré de remplir d'émotions une vie creuse $^{49} »$.

La conversation entre les amies est le vecteur privilégié de ces réflexions sur le fonctionnement social et individuel. Dans leur quête de compréhension de ce qu'elles sont, elles commencent en effet par démonter ce qu'elles voient se développer chez d'autres et qu'elles récusent. Elsa explique à Raquel dans le chapitre 7 que l'éducation par le mensonge se fait dès l'enfance et se transforme en frustration à l'âge adulte:

le contraire de ce qu'on nous a appris, à toi et à moi, quand nous étions petites (...) c'est pour cela qu'échoue la majorité des couples : parce qu'on attend qu'ils agissent selon ce qu'on nous a appris $^{50}$

30 L'apprentissage dont il est question associe éducation et religion. Celle-ci apparaît sous la forme d'un enseignement dispensé par des religieuses dans des écoles privées payantes ${ }^{51}$. Sur le fond, l'essentiel de cette éducation consiste à apprendre à l'enfant à différencier ce que certains considèrent comme le bien et le mal ${ }^{52}$. La critique consiste à montrer que l'enjeu de cette éducation est durable, profond et pervers ${ }^{53}$. Mais il s'agit dans ce roman d'encourager à s'y opposer. Pour cela la voix narrative, quelle qu'elle soit, s'attache à faire apparaitre qu'il peut être limité, qu'on le subit mais qu'on peut refuser de s'y soumettre. Il est question de perspective parce qu'en réalité cela n'empêche pas de commettre les "péchés mortels " qu'on souhaite, mais on le fait en conscience. Ironiquement Elsa 
déclare que "d'une certaine manière, elle est toujours catholique ${ }^{54}$ " parce qu'elle sait qu'elle fait mal selon les canons de la religion dans laquelle elle a été élevée.

31 L'incitation au dépassement de soi par un possible changement de collectif trouve sa matérialisation dans le personnage de Maria. Dans le chapitre 4, l'étape antérieure est évoquée avec sévérité comme celle d'« une complaisance ignorante et bovine ». Elle est liée au non-questionnement, à la répétition, marquée par le champ lexical de la platitude et du mirage du bonheur ${ }^{55}$, caractérisée par « le goût amer de la routine, la dégradation invisible du quotidien ${ }^{56}$ ». Susi, dans le chapitre 16 , constate avec la même image, c'est-àdire en dégradant par animalisation et indifférenciation de ses membres le collectif de l' exogroupe, qu'elle est entourée du "troupeau de la majorité, solide et têtu ${ }^{57}$ ", mais elle aussi en est sortie.

On le voit, le stéréotype lié à l'endogroupe comprend deux axes, l'identité sociale et l'identité personnelle, cette dernière étant à la fois professionnelle, sexuelle, générique ou de classe. Ce collectif de référence, auquel se sentent appartenir le sujet producteur du récit et les différences instances narratrices, s'auto-attribue une certaine identité qu'il est en train de chercher à affirmer. Face à lui se trouve l'exogroupe, cet autre collectif sur lequel les premiers projettent des stéréotypes pour catégoriser les autres sujets. La dialectique que l'on observe ici est celle, habituelle, du " favoritisme en ce qui concerne l' endogroupe » et de "l'homogénéisation en ce qui concerne l'exogroupe ». Les membres de ce dernier ne font l'objet dans ce roman d'aucune marque d'identité personnelle et l'indifférenciation mène à leur « désindividualisation ». Il s'agit d'un schème collectif figé, sans voix, d'une représentation commune, sans appel.

33 Le chapitre 16, final, fait se rejoindre pour la seule fois ces quatre femmes aux itinéraires différents, mais aux traits semblables et il les oppose une dernière fois au groupe des « autres ». Jusqu'à ce moment-là, le lecteur devait reconstruire lui-même les traits du stéréotype, comme on l'a vu ci-dessus. A partir de la page 383, s'effectue la synthèse. Le chapitre fonctionne sur le va-et-vient systématique entre les deux groupes, la comparaison, la tentation puis le rejet, la mise à distance, reprenant les différents éléments apparus séparément.

34 Le stéréotype de genre ou d'âge ne hiérarchise pas la femme et l'homme par une différence entre don et doña, señorita et señorito, mais les femmes entre elles, avec l'accent de «señorita de dinero" (p.392), de demoiselle de bonne famille, ce qui marque la position sociale. Il n'y a pas de patronyme, ni de "señora de" qui marquerait la possession $^{58}$, mais des filles qui ont un nom double, figurant l'appartenance généalogique ou un nom d'épouse accolé, dans le double sens entretenu de ce "nom composé » (« apellido compuesto » p.393).

35 Dans la bouche d'un des personnages, Raquel, sous une forme à peine différente, revient l'idée du titre ( las demás »): « elle croyait que Maria était différente, une fille de chez les autres ${ }^{59} "$, en italique dans le texte. Avant le rejet, s'insinue la tentation de rejoindre ce groupe, d'être pour une fois du côté des autres, où les individus sont vus, de façon hyperbolique, comme protégés en tous points, matériellement et socialement:

la très bonne famille, les études achevées avec des notes excellentes, l'appartement au centre, le nom double, la réputation sans tâche (...) un miroir de vertus, une âme rangée, soignée et docile, aussi bien à l'intérieur qu'à l'extérieur, tellement courtoise et délicate dans sa brillante médiocritét ${ }^{\text {s. }}$. 
C'est ce terme de «médiocrité » dans son sens premier de "niveau moyen », d' « aurea mediocritas » refusée, qui marque la position sur l'échelle de valeurs dans le jeu entre les stéréotypes. C'est aussi lui qui indique que les personnages vont résister à la tentation parce que les filles de cette catégorie ne sont pas complètes, parce qu'elles sont « en noir et blanc» (p.394). Face à ce portrait aux couleurs limitées, on retrouve visuellement l'autre catégorie de femmes, aux couleurs vives et variées ${ }^{61}$. A la place d'un patronyme, ces filles se regroupent sous un hétéronyme qui, par association métaphorique, fait référence à la femme dangereuse. C'est la "marimacho », qu'on appelle ici virago ${ }^{62}$. Ce terme revêt a priori une connotation négative parce que la femme en question ne répond pas à l'idéal féminin défendu par la tradition androcentrique. Comme certains groupes féministes avaient déjà révisé ce terme en l'utilisant comme nom (ex: Virago Press à Londres), ici, il est nettement revendiqué et associé à la notion de combativité puisqu'il constitue le titre du chapitre. En outre, les quatre personnages féminins qui évoluent sous ce titre vont personnifier l'enjeu identitaire. Si des relations de nature lesbienne se tissent dans une boîte de nuit, elles marquent à présent la différence, la volonté de se démarquer : "Susi se demande pourquoi, quand toutes les têtes dansent à l'unisson, elle est prise de cette tentation aiguë de détonner ${ }^{63} »$.

37 En même temps est crié le besoin de n'être pas seule, de pouvoir s'associer à un groupe de gens semblables, afin de pouvoir "se voir dans un miroir humain" (p.390) pour parvenir à exister, en dépit de la sensation d'être « bizarres » et « inadaptées » (p.59) dans un monde fait et pensé par les autres. Parce qu'à l'extérieur du groupe aussi, se trouve l'anonymat, par le fait duquel personne ne se préoccupe de la solitude, de l'angoisse, des souvenirs, des délires et des frustrations de toutes ces femmes en quête d'identité, et non de ce bonheur que poursuivent les autres, sans jamais l'atteindre.

Nous avons mis en évidence tout un jeu sur les stéréotypes. Ceux-ci sont, dans l'œuvre, voulus et instrumentalisés, et à plusieurs reprises la voix narrative témoigne de la conscience du décalage entre les méthodes qui opèrent dans la fiction et la réalité :

dans la vie réelle les lieux communs ne fonctionnent pas, l'absolu et les exemples déterminants n'existent pas, parce que la majorité des personnes sont trop compliquées pour que leurs réactions puissent être expliquées en fonction d'émotions prédéterminées ${ }^{64}$

39 Au terme de cette analyse de Nosotras que no somos como las demás qui a montré comment se mettait en place le jeu de stéréotype contre stéréotype, se confirme l'importance du rôle du lecteur à qui il revient de "dégager un schème abstrait à partir de données souvent indirectes, éparses ${ }^{65}$ » pour mettre en évidence le sens profond au-delà de l'anecdote. Il s'agit véritablement d'une activité de déchiffrement qui consiste à retrouver les attributs non pas ici d'un seul groupe, mais de deux, à partir de formulations variées. Le stéréotype est en fait une construction de lecture ${ }^{66}$ dont l'activation dépend de la capacité d'abstraction du lecteur et de la culture dans laquelle il baigne.

A l'inverse, Iser et Eco ont montré l'importance du stéréotype dans le processus de lecture, l'oeuvre littéraire le stimulant autant par ce qu'elle dit que par ce qu'elle ne dit pas, strates d'implicites que le lecteur doit activer ${ }^{67} »$. Il existe une certaine prévisibilité sur la base de scénarios intertextuels (cf les frame de Eco) en particulier issus de la littérature féministe. En outre « le scénario motif » de la femme libérée se superpose aux "scénarios situationnels » dans les scènes de sexe ou les évocations de la déroute et de la solitude... Pour Jean-Louis Dufays ${ }^{68}$, apprendre à lire c'est apprendre à repérer les 
" constellations figées, des schèmes partagés par une communauté donnée ». Lucia Etxebarria guide le lecteur sur ce chemin ; elle explicite les stéréotypes justement en les opposant tout au long de l'oeuvre.

41 Pour Jauss, la qualité d'une œuvre littéraire vient de sa capacité à infléchir, voire bouleverser, les idées toutes faites car c'est justement l'apanage de la paralittérature, ou littérature de masse, que de se nourrir exclusivement de formes stéréotypées ${ }^{69}$. Lucía Etxebarria a souvent vu taxer ses œuvres à succès comme Amor, curiosidad, Prozac y dudas, de littérature « light ».

42 C'est peut-être dans le dispositif énonciatif particulier autour de l'opposition entre stéréotypes que réside l'originalité de cette œuvre. Elle procède à une construction identitaire dans un texte dont l'esthétique propre permet de se dégager, en qualifiant explicitement d'idées reçues la conclusion du procès. Cela installe une distance critique, sans laquelle il pourrait y avoir condamnation esthétique ou idéologique, qui resémantise le stéréotype en quelque sorte dans son rapport à l'imaginaire social.

\section{NOTES}

1. Lucía Etxebarria, Nosotras que no somos como las demás, Barcelone, 2006, Edition Destino, coll. Booket.

2. Voir les travaux de Paul Ricoeur Soi-même comme un autre, Paris, Seuil, 1990

3. « como si se tratara de una novela », p.11, Nosotras ...,id. Pour que les enjeux des stéréotypes qui résident au niveau linguistique (double-sens, sous-entendus, spécificités de l'espagnol, etc) soit perceptibles, nous faisons figurer le texte original en note ; pour les références de l'édition citée, voir n1. Les traductions des citations utilisées sont de $C$. Di Benedetto.

4. « en el principio era un libro de relatos... », p.10, ibid..

5. « infinitas Marías que componían todo el espectro definible de las personalidades femeninas (...) a solas consigo misma, o con sus nosotras », p.103, ibid..

6. Ruth Amossy, Stéréotypes et clichés : langue, discours, société, Paris, 2005, ed.A.Colon, coll.128, p.65-66.

7. Le titre, Visio Smaradigna est construit à partir de l'expression latine Tabula Smaragdina, La Table d'émeraude. Wikipedia, que le grand public peut facilement consulter pour comprendre ce titre, apparemment crypté, explique qu'il s'agit d'un document seulement accessible à de rares initiés, « un des textes les plus fameux de la littérature alchimique et hermétique. Selon la légende, il présente l'enseignement de Hermès Trismégiste, fondateur mythique de l'alchimie, et aurait été retrouvé dans son tombeau, gravé sur une tablette d'émeraude. C'est un texte très court, composé de formules allégoriques et obscures, dont la célèbre correspondance entre le macrocosme et le microcosme : «Ce qui est en bas est comme ce qui est en haut, et ce qui est en haut est comme ce qui est en bas ». Traduit de l'arabe au XII ${ }^{\circ}$ s., il est commenté et glosé par les alchimistes au Moyen Âge et surtout à la Renaissance. La plus ancienne version connue se trouve en appendice d'un traité arabe du VIe siècle. ». 
8. « la humillación de visitar una sex-shop (territorio masculino, por. Lo general) » p.16, Nosotras..., id.

9. « El criterio de selección de los protagonistas de aquellos vídeos coincidía con unos cánones descaradamente norteamericanos que Raquel dudaba mucho se correspondieran con los gustos francamente patrios de Elsa » p.172, ibid.

10. « No son como los demás... », p7, ibid.

11. « los roles que adoptamos », p.8, ibid.

12. « nuestra identidad sexual -lo que sentimos que significa ser hombre o mujer -», p.8, ibid.

13. « elaboración asocial de un rasgo cultural » p7, ibid.

14. Henri Tajfel et J.C. Turner, (1979) An integrative theory of intergroup conflict. In: W.G. Austin and S. WorcheL (eds) The Social Psychology of Intergroup Relations, Monterey, Calif.: Brooks/Cole.

15. « Algunas mujeres no nos conformamos », « no nos gusta », « estamos hartas », p.9, Nosotras..., id.

16. « algunas mujeres protestamos », p.10, ibid.

17. Ruth Amossy, cite ainsi les résultats de recherches menées à bien par des psychologues sociaux américains en 1981 et qui établissent que la première croyance stéréotypée concernant les femmes serait leur «soumission ». Les idées reçues- sémiologie du stéréotype, Paris, Nathan, 1991 (p170).

18. On sait que pour lutter contre certains stéréotypes nationaux présents, en particulier, dans les manuels scolaires en Espagne, il a fallu en opposer d'autres, par la même voie, de caractère paneuropéen dans le cadre de l'entrée du pays dans la Communauté européenne. Cela est visible dès les années 90 . Voir Une idée fausse est un fait vrai, direction Jean-Noël Jeanneney, Paris, Ed. Odile Jacob, 2000 (p59).

19. « si estás en el segundo serás el centro de una polémica constante. Medio mundo te odiará, te crucificará por la menor de tus acciones y te convertirá en el objetivo de sus temores, sus odios y sus desprecios » p.295, Nosotras..., id.

20. Antoinette Fouque, Il y a 2 sexes- Essais de féminologie, Paris, Gallimard, coll. Le Débat, 2004. « Tant qu'il y aura des femmes », p.279-80.

21. «Las chicas fáciles, las zorras, las putas, las robamaridos, las que no cuidamos niños, las que entramos y salimos cuando y por donde nos viene en gana... Las que no llevamos anillo con una fecha por dentro », p.295, Nosotras..., id.

22. Ruth Amossy, Stéréotypes et clichés, id., p.66.

23. Roland Barthes en 1975, cité par Ruth Amossy, Stéréotypes et clichés, id., p.63.

24. Ruth Amossy, ibid, p.64.

25. Ruth Amossy, id..

26. « la búsqueda merece, en sí misma, la pena », p.205, Nosotras..., id.

27. Pour analyser les fonctions spécifiques des préjugés et stéréotypes dans les communications entre groupes, on peut distinguer trois types de représentations : les représentations induites, les représentations justificatrices et les représentations anticipatrices (Doise, 1979). En parlant de représentations induites, on signifie qu'elles sont le reflet des interrelations présentes ou passées entre les groupes. Avec les représentations justificatrices, on aborde la fonction le plus souvent dégagée par l'observation et l'expérimentation. Mais les préjugés peuvent avoir aussi une fonction anticipatrice ; ils préparent au niveau imaginaire la situation qu'un groupe veut atteindre ou l'action qu'il souhaite entreprendre.

28. « la experiencia más reveladora de su vida » p.102, ibid.. 
29. « se acaba amando aquello que se teme », p.182, ibid.

30. « en el amor, como en la bolsa, cuanto más inviertes, más pierdes », p.182, ibid.

31. « tan inexplicable como el sexo de los ángeles », p.177, ibid..

32. « oraciones heréticas, ritos profanos, sacrílegos bautismos de sudor y jadeos », p.99, ibid.

33. « la suma de dos y uno no era tres. María había entendido, por fin, por qué la Trinidad se entiende como un Uno ", p.99, ibid.

34. « entrega de su carne para hacerla renacer, para desafíar su condición de mortal ", p.100, ibid.

35. " el uno contra el otro reinventaron la inicial explosión de la que brotó el cosmos », p.105, ibid.

36. « siempre lo mismo, siempre distinto, como cada día, como la vida », p.105, ibid.

37. « algo se le desinfló a Eduardo por dentro al comprender que, en realidad, ella le había estado dirigiendo todo el rato », p.342, ibid.

38. « empezaba a comprender lo sencilla y segura que puede aparecer la depravación cuando se ha reunido el valor para empezar $»$, p.177, ibid.

39. « nube empática del hachís » p.79, « sílfide esquelética... los ojos drogados entornados » p.81, ibid.

40. Dirección General de la Mujer - Consejería de Trabajo, Arrinconando estéreotipos en los medios de comunicación y en la publicidad, Areste, Comunidad de Madrid, 2003, p.1/35.

41. « jamás conseguiría equilibrar (... ) las competidoras exigencias de la necesidad de querese a sí misma y la de querer a otros, la angustia de opresión y la angustia de abandono », p.195, Nosotras..., id.

42. " a él lo vestían de azul y a ella de rosa », p.37, ibid.

43. Les rôles sont établis dans la famille ; le frère est le " prince détrôné » quand arrive la petite fille (p.37) mais il reprend sa place, puis rentre dans le rang et devient : « le projet d'un monsieur quelconque, portant cravate du lundi au vendredi... comme il se devait » (« un proyecto de señor cualquiera que se encorbata de lunes a viernes... como convenía », p.51, ibid.

44. « ils les eurent (les enfants) pour la même raison qu'ils se marièrent : parce que c'était ce qu'il fallait faire » (« los tuvieron por lo mismo que se casaron : porque era lo que había que hacer $»)$, p.41, ibid.

45. « tuvo que casarse con la madre para poder acostarse con ella (porque llegó virgen al matrimonio (...) el único gesto del que se podía sentir orgullosa en su tediosa y estéril vida) », p.41-42, ibid.

46. Le sexe ainsi vécu demeure une contrainte pour la mère qui « haïssait le sexe (...) et disait qu'il ne fallait pas parler de cette chose-là » (« odiaba al sexo... decía que de eso no se hablaba »), p.43, ibid.

47. « una gimnástica amatoria y lanzamiento de vajilas » p.174, ibid.

48. « qué es la familia, qué es la infancia, al fin y al cabo, sino un tortuoso entrenamiento al masoquismo », p.182, ibid.

49. « una adicción, un enganche a la angustia, una desesperada necesidad de llenar de emociones una vida hueca », p.183, ibid.

50. « al contrario de lo que a ti y a mí nos han enseñado de pequeñas... por eso fallan la mayoría de las parejas : porque esperamos que actúen según nos han enseñado », p.177-78, ibid.

51. « educación de colegio de pago », p.83 ; « colegio de monjas », p.173, ibid. 
52. « de pequeña me enseñaron la diferencia entre el bien y el mal », ; « sé cuando hago mal », p.185, ibid.

53. Le paradoxe est que, comme il est établi dans Les liaisons dangereuses, « le chemin le plus direct vers la perversion passe par l'innocence absolue » (« el camino más directo a la perversión pasa por la inocencia absoluta »), p.199, ibid. La mise en pratique de cette théorie se fait avec l'exemple de María qui avoue n'avoir connu aucune éducation sexuelle, à part ses propres expériences, et donc considéra toujours que son partenaire en savait plus qu'elle sur ce qui était normal, d'où une latitude énorme laissée à l'autre, latitude dont la cause est l'ignorance.

54. « en cierto modo, sigo siendo católica », p.186, ibid.

55. « ... comme elle l'avait été à peine six mois auparavant, lorsque la vie était tiède et lisse, calme, et chaque jour dans sa béatitude, paraissait identique au précédent et au suivant » (« complacencia ignorante y bovina, que había sido apenas seis meses antes, cuando la vida era tibia y plana, sosegada, y cada día, en su beatitud, parecía idéntico al anterior y al siguiente »), p.103, ibid.

56. « amargo sabor de la rutina, el deterioro invisible de la cotidianidad», p.90, ibid.

57. « el sólido y terco rebaño de la mayoría », p.387, ibid.

58. Pedro A Fuertes Olivera, Mujer, lenguaje y sociedad - Los estereotipos de género en inglés y en español, 1992, Univ. de Valladolid, chap.VIII.

59. « ella creía que María era distinta, una chica de las otras », p.391, Nosotras..., id.

60. « la buenísima familia, la carrera acabada con notas excelentes, el apartamento céntrico, el apellido compuesto, la reputación intachable » (p.393) ; « espejo de virtudes, un alma ordenada, pulcra y dócil, tanto por dentro como por fuera, tan cortés y delicada en su brillante medianía », p.394, ibid.

61. « comme si quelqu'un avait accentué leurs couleurs » (« como si alguien hubiera acentuado sus colores »), p.394, ibid.

62. Pedro A. Fuertes Olivera, Mujer, lenguaje y sociedad - Los estereotipos de género, id.. 63. «Susi se pregunta por qué, cuando todas las cabezas bailan al unísono, a ella le acomete esa punzante tentación de desafinar », p 387, Nosotras..., id.

64. « en la vida real los lugares comunes no funcionan, no existe el absoluto ni los casos determinantes, porque la mayoría de las personas son demasiado complicadas como para que sus reacciones puedan explicarse en función de unas emociones predeterminadas ", p.177, ibid.

65. Ruth Amossy, Stéréotypes et clichés, id., p.73.

66. Ruth Amosy, ibid, p.21-22.

67. Ruth Amosy, ibid, p.75

68. J_Louis Dufays, Stéréotype et lecture, Liège, Ed. Mardaga, 1994,

69. Voir Pierre Bourdieu qui parle de « champ de grande production » dans lequel il faut éviter les ruptures de communication pour assurer la réception adéquate. 


\section{RÉSUMÉS}

Ce roman de Lucía Etxebarria, « Nous, qui ne sommes pas comme toutes les autres ", s'inscrit dans le concept d'altérité qui guide la lecture en installant comme horizon d'attente celui de l'affirmation identitaire. Sont figurés deux groupes antagonistes, de femmes, mais pas seulement, dont les traits apparaissent progressivement par un double décryptage autour de la notion de stéréotype, ses enjeux et ses formes dans la narration. De la mise en regard de ces collectifs, que des caractéristiques volontairement marquées rendent potentiellement identifiables, découle le sens de l'oeuvre, sorte de positionnement axiologique dans l'Espagne du troisième millénaire. Le dispositif énonciatif particulier autour de l'opposition entre stéréotypes constitue l'enjeu de cette œuvre, lui permettant de dépasser le niveau qui associe stéréotype à littérature de masse. En découvrant explicitement les idées reçues de part et d'autre, et en en jouant pour les révéler, le roman installe une distance critique qui repousse la condamnation esthétique ou idéologique et resémantise le stéréotype dans son rapport à l'imaginaire social.

\section{INDEX}

Mots-clés : axiologie, enjeu esthétique, femmes, idée reçue, imaginaire social, société, stéréotype

Index chronologique : XXe - XXIe siècles 